\title{
Performance Evaluation Framework for Vertical Handoff Algorithms in Heterogeneous Networks
}

\author{
Ahmed H. Zahran and Ben Liang \\ Department of Electrical and Computer Engineering \\ University of Toronto, Toronto, Canada \\ Email: zahran, liang@comm.utoronto.ca
}

\begin{abstract}
The next generation (4G) wireless networks is envisioned as a convergence of different wireless access technologies providing the user with the best anywhere anytime connection and improving the system resource utilization. The integration of Wireless Local Area Network (WLAN) hotspots and third generation (3G) cellular network has recently received much attention. While the 3G-network will provide global coverage with low data-rate service, the WLAN will provide high data-rate service within the hotspots. Although increasing the underlay network utilization is expected to increase the user available bandwidth, it may violate the Quality-of-Service $(\mathrm{QoS})$ requirements of the active real-time applications. Hence, achieving seamless handoff between different wireless technologies, known as vertical handoff (VHO), is a major challenge for 4G-system implementation. Several factors should be considered to realize an application transparent handoff such as application QoS requirements and handoff delay. In this paper, we present a novel framework to evaluate the VHO algorithm design impact on system resource utilization and user perceived QoS. We used this framework to compare the performance of two different VHO algorithms. The results show a very good match between simulation and analytical results. In addition, it clarifies the tradeoff between achieving high resource utilization and satisfying user QoS expectations.
\end{abstract}

Keywords Vertical handoff, heterogeneous networks, performance evaluation.

\section{INTRODUCTION}

Wireless technology provides different alternatives for its users that vary in terms of coverage, bandwidths, delays, security, and cost of both implementation and service. The next generation wireless networking $(4 \mathrm{G})$ is envisioned as a convergence of different wireless access technologies keeping the user connected to the best available access network [1]. Additionally, this convergence is expected to improve the preinstalled infrastructure utilization. In the wireless networks hierarchy, overlay networks usually provide low data-rate expensive global services, while underlay networks provide cheaper high data-rate services within hotspots. Hence, overlay networks are preferable for high-mobility users with moderate or low traffic demand, while the underlay networks are recommended for larger users densities with higher traffic demands. In this sense, different wireless access technologies are complementary.

The integration of wireless local area networks (WLAN) and third generation $(3 \mathrm{G})$ cellular networks has been recently a subject of great interest [2]-[4]. On one hand, 3G networks will provide global coverage at limited data rates; on the other hand, WLANs will provide higher data rates within

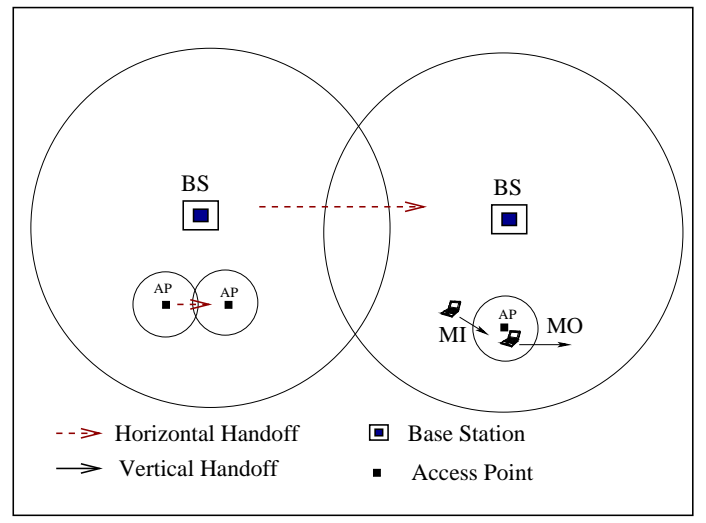

Fig. 1: Handoff types in $4 \mathrm{G}$ model

hotspots. The integration process is propelled by the interest in bandwidth consuming applications and the clustered nature of traffic due to its concentration in public areas such as hotels, cafe-shops, and airports. Hence, $3 \mathrm{G}$ cellular service providers will be able to offload such heavy traffic to WLANs, saving the 3G-network precious wireless resources for users located outside WLAN coverage. As a result, wireless Internet service providers will have a new revenue source and will improve the utilization of their pre-installed infrastructure. Meanwhile, network subscribers will enjoy the best features of both technologies including universal coverage, larger bandwidth, and lower cost of combined services on one bill.

However, several service and system design issues must be addressed before practical implementation [5]. Most of these issues are highly correlated and cannot be addressed separately. For example, the system architecture has a great impact on the used protocols and standards [6] and will consequently affect other issues such as mobility management and quality-of-service (QoS). This paper addresses vertical handoff (VHO), handoff between heterogeneous networks, as one of the main system design challenges. Handoff is generally defined as a process in which a Mobile terminal (MT) utilized resources are transferred as it changes its point of attachment. The network heterogeneity results in an asymmetric handoff process as shown in Figure 1. Consequently, two different vertical handoff scenarios are defined: Moving Out of the preferred network (MO) and Moving In to the preferred network (MI) [7], where the preferred network is usually the 
underlay network that provides better and cheaper service. Consequently, extending the utilization of the WLAN, as long as it provides the user with satisfactory performance, is one of the main considerations of vertical handoff algorithm design.

In this paper, a novel performance evaluation framework for a vertical handoff algorithms is presented. This framework considers different metrics including the number of handoffs, the integrated network resource utilization, and some QoS parameters of the active applications during vertical handoff. The rest of the paper is organized as follow. In Section II, we explain the requirements of vertical handoff algorithm that inspires the proposed evaluation model. The performance evaluation framework is introduced in section III. Section IV shows the obtained results using this framework. Finally, the conclusions are presented in section $\mathrm{V}$.

\section{Vertical handoff Algorithm}

The handoff process has two main phases, namely: decision phase and execution phase. During the execution phase, signaling messages are transmitted to direct the incoming traffic toward the new attachment point. In our $4 \mathrm{G}$ model, two major approaches are proposed for signaling: the infrastructure-based approach and the end-to-end approach. The former introduces new nodes such as special agents to the network, while the latter pushes the network intelligence toward the endhosts. Mobile-IP (MIP) and session initiation protocol (SIP) are examples of these approaches respectively. It is worth mentioning that the location management mechanism impact on the decision phase is limited to handoff signaling delay.

In homogeneous systems, handoff algorithms consider several rules and mechanisms for handoff decision [8] using one or more of the following strategies: thresholds, hysteresis, timers, and different window shapes/sizes. In the converged model, the MI and MO scenarios will have different handoff mechanisms. For MI, WLAN availability is crucial; however, other factors such as WLAN condition and user preferences can be considered in a policy-based MI decision [9]. On the other hand, extending the utilization of the underlay network is one of the main requirements in MO strategy to improve system resource utilization and increasing user available bandwidth. This requirement motivates considering a dwelling timer as a MO strategy as in [10] and [11]. In this approach, the MT persists on using the WLAN in a bad condition for the entire dwelling timer duration. This strategy may be effective for non-real-time applications because using the WLAN for a short time may be better than using the slower $3 \mathrm{G}$ networks for long time. On the other hand, the dwelling timer strategy may result in service interruption periods for active real-time sessions due to the serious shadow fading effect on the WLAN Received Signal Strength $(R S S)$ near WLANs' edge. In [12], the authors introduce a lifetime-based algorithm considering both active application and location management delay requirements. This algorithm estimates the WLAN availability duration, i.e. lifetime, considering the active real-time application QoS requirements. The MT WLAN lifetime is estimated as [12]

$$
E L[k]=\frac{\overline{R S S}[k]-A S S T}{S[k]},
$$

where $\overline{R S S}[k]$ is an averaged version of the $R S S, A S S T$ is a threshold chosen to satisfy the requirements of the active applications, and $S[k]$ represents the $R S S$ decay rate. Additionally, it proactively performs the MO when the WLAN $R S S$ falls below a predefined MO threshold and the estimated lifetime is equal to the required handoff-signaling delay.

Although extending the WLAN utilization is a common approach in the aforementioned algorithms, the critical fading impact near the cell edges may result in severe degradation in the user perceived QoS. This phenomenon results in a tradeoff between improving the system resource utilization and satisfying the user QoS requirements. Hence, we develop our handoff performance evaluation framework considering all these issues. In order to consider efficient resource utilization, we used WLAN utilization as a performance metric. In addition, the user available bandwidth and packet delay are the main QoS considered metrics. Furthermore, the number of handoffs is considered as a main measure for satisfactory handoff algorithm performance. To sum up, the main metrics that we are interested in are:

- the number of unnecessary HOs,

- wireless resources utilization,

- user available bandwidth, and

- user packet delay.

In the next section, we present our analytical framework according to the aforementioned metrics.

\section{Performance evaluation framework}

\section{A. System model}

In our model, the overlapping of $3 \mathrm{G}$ cellular network and WLAN is assumed, using MIP for location management. It is worth mentioning that a special WLAN gateway is required to integrate both networks services [3], [6]. This gateway performs several tasks including MIP agents, Authentication Authorization and Accounting (AAA) server, and dynamic stateful firewall service. In addition, it can manage class-based QoS operations within WLANs; however, supporting end-toend QoS requires other mechanisms such as differentiated services implementation over the entire network path. For purpose of illustration, we later assume the converged networks are CDMA2000 and IEEE802.11a based WLAN.

The MT is equipped with dual interfaces allowing communication with both networks. However, the MT connects to one network only at a time because MIP provides only one tunnel. Furthermore, a multi-interface mobility client software is installed on the MT. This client software periodically scans the available interfaces for the observed RSS from different networks, intelligently selects the best access network according to the adopted VHO algorithm, and triggers signaling required for MIP and link-level associations including PPP connections for $3 \mathrm{G}$ networks and LAN association for WLAN access points. 


\section{B. Performance Evaluation Analysis Framework}

In order to evaluate the handoff algorithm performance, several models should be considered such as channel model and MT mobility model. In our framework, a log-linear path loss channel propagation model with shadow fading is assumed. The RSS is expressed as a function of the distance between the MT and the access point $(d)$ as [13]

$$
R S S(d)=P_{T}-L-10 n \log (d)+f(\mu, \sigma) d B m,
$$

where $P_{T}$ is the transmitted power, $L$ is a constant signal power loss, $n$ is the path loss exponent that usually has values between $2-4$, and $f(\mu, \sigma)$ represents shadow fading modeled as zero mean Gaussian random variable with mean $\mu$ and standard deviation $\sigma$ with values between 6-12 $\mathrm{dB}$ depending on the environment. Consequently, different averaging mechanisms are used to decrease the effect of signal variation due to these fluctuations. In the discrete problem, the WLAN RSS is sampled every $T_{\text {sampling }}$; hence, it is expressed as

$$
R S S[k]=\mu_{R S S}[k]+N[k] d B m,
$$

where $k$ is the time index, $\mu_{R S S}[k]=P_{T}-L-10 n \log (d[k]), d[k]$ is the distance between the MT and the WLAN Access Point (AP) at instant $k$, and $N[k]=f(\mu, \sigma)$. Since the distance from the AP is the unique parameter that affect the $R S S$, a simple mobility model will be sufficient for performance evaluation of the handoff algorithm. Furthermore, within the time-scale of handoff, a MT is unlikely to change its velocity. Hence, we consider a MT moving away from the AP at a constant speed $V \mathrm{~m} / \mathrm{s}$.

The transition probabilities calculation is based on recursive handoff probabilities computation as in [14]. In the integrated model, the following probabilities are required for the analysis of any handoff algorithm

- $P_{W}[k]: \operatorname{Pr}\{\mathrm{MT}$ is associated with the WLAN at instant $k\}$

- $P_{C}[k]: \operatorname{Pr}\{\mathrm{MT}$ is associated with the $3 \mathrm{G}$ network at instant $k\}$

- $P_{W \mid C}[k]: \operatorname{Pr}\{\mathrm{MT}$ will associate itself with the WLAN at instant $k$ given that it is associated with the cellular network at instant $k-1\}$

- $P_{C \mid W}[k]: \operatorname{Pr}\{$ MT will associate itself with the $3 \mathrm{G}$ network at instant $k$ given that it is associated with the WLAN at instant $k-1$ \}

In our model, the MT is assumed to be attached to the WLAN at the beginning; hence, $P_{W}[0]=1$ and $P_{C}[0]=0$. Consequently, $P_{W}[k]$ and $P_{C}[k]$ can be calculated recursively as follows.

$$
\begin{aligned}
& P_{W}[k+1]=P_{W \mid C}[k+1] P_{C}[k]+\left(1-P_{C \mid W}[k+1]\right) P_{W}[k], \\
& P_{C}[k+1]=P_{C \mid W}[k+1] P_{W}[k]+\left(1-P_{W \mid C}[k+1]\right) P_{C}[k],
\end{aligned}
$$

where the conditional probabilities $P_{C \mid W}[k+1]$ and $P_{W \mid C}[k+$ 1] depend on the handoff algorithm initiation strategy. Then, these resulting transition probabilities are used to calculate the performance metrics as follows.

The number of handoffs, denoted $N_{H O}$, is calculated as the sum of MOs and MIs between WLANs and 3G networks.

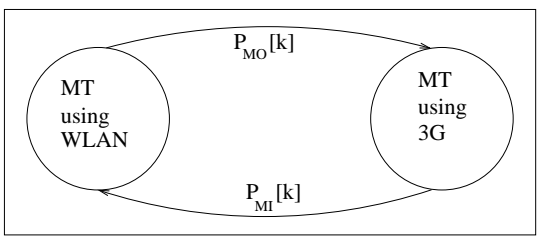

Fig. 2: VHO Markov chain model

Hence, $N_{H O}$ is a random variable that depends on the instantaneous move out/in probabilities that can be calculated by

$$
\begin{aligned}
P_{M O}[k+1] & =P_{C \mid W}[k+1] P_{W}[k], \\
P_{M I}[k+1] & =P_{W \mid C}[k+1] P_{C}[k] .
\end{aligned}
$$

The MT movement between the two networks can be modeled by a two-state non-homogeneous Markov chain. Each state represents the network with which the MT is associated. The transition probabilities are $P_{M O}[k]$ and $P_{M I}[k]$ as shown in Figure 2. Hence, by using binary impulse rewards for the state transitions as shown in [15], we calculate the average accumulated reward for MO and MI transitions, which are equivalent to the expected number of MOs, $E\left\{N_{M O}\right\}$, and the expected number of MIs, $E\left\{N_{M I}\right\}$ respectively. Hence, the expected number of handoffs, $E\left\{N_{H O}\right\}$, can be calculated by

$$
\begin{aligned}
E\left\{N_{H O}\right\} & =E\left\{N_{M O}\right\}+E\left\{N_{M I}\right\} \\
& =\sum_{k=1}^{k_{\max }}\left(P_{M O}[k]+P_{M I}[k]\right) .
\end{aligned}
$$

In order to have an appropriate definition for the WLAN utilization and consider the impact of service degradation near the WLAN edge, WLANs are defined to be in one of two states: WLAN Up and WLAN Down. In former, the MT RSS is above the interface sensitivity level, $\alpha$, while the latter is the reverse case. Hence, we define the WLAN channel state at the MT as a binary random variable $X[k]$, so that $X[k]=1$ represents the WLAN Up state and $X[k]=0$ represents the WLAN Down state. Consequently, we have

$$
X[k]=\left\{\begin{array}{ll}
1, & R S S[k] \geq \alpha \\
0, & R S S[k]<\alpha
\end{array} .\right.
$$

Let $p[k]=\operatorname{Pr}\{X[k]=1\}$. Clearly

$$
\begin{aligned}
p[k] & =\operatorname{Pr}\{R S S[k]>\alpha\} \\
& =Q\left(\frac{\alpha-\mu[k]}{\sigma}\right)
\end{aligned}
$$

where $Q(x)$ is the complementary error function as $\frac{1}{\sqrt{2 \pi}} \int_{x}^{\infty} e^{-y^{2} / 2} d y$.

Using this model, the WLAN utilization is defined as the percentage of the MT captured WLAN Up states during WLAN usage over the total WLAN Up states within its coverage area. It is worth mentioning that capturing all the up states implies handing off outside the WLAN which may lead to severe QoS degradation and annoying interruption periods. In order to calculate the WLAN utilization, we define the 
average MO distance as the average distance between the MT and the WLAN AP at which the MT performs the MO using a certain algorithm, as a measure for the MT WLAN usage duration. The average MO distance can be estimated as

$$
\mu_{d}=\sum_{k=1}^{k_{\max }} \overline{P_{M O}}[k] d[k],
$$

where $\overline{P_{M O}}$ is a scaled version of $P_{M O}$ to represent a valid PDF within interval $\left[1, k_{\max }\right]$, and $k_{\max }$ represents the time index at which the MT reaches the WLAN edge determined by the planed coverage area.

For fair realistic comparison, we compare the performance of the handoff algorithms within the transition region, in which the RSS starts to oscillate around the interface sensitivity. The determination of transition region boundary is an old complex level crossing problem that is analytically tractable for only a few simple cases and is usually solved numerically for complex cases. Hence, the transition region starting point is obtained from our simulations and then used in the analysis. The expected beneficial WLAN utilization can be then determined by the expected captured up time $\mu_{T_{C}}$ of a handoff algorithm, which can be defined as

$$
\mu_{T_{C}}=E\left\{T_{C}\right\}=E\left\{\sum_{k=k_{\text {start }}}^{k_{M O}} X[k]\right\},
$$

where $k_{\text {start }}$ represents an approximate estimate for the transition region start, and $k_{M O}$ is a random variable that represents the time index for the MO distance. Clearly, this summation can not be directly computed due to the varying statistics of $X[k]$ and the correlation between the summation upper bound $k_{M O}$ and $X[k]$. Hence, we consider a first order approximation by using the average MO distance index $\overline{k_{M O}}$ as an upper bound for the summation. Consequently, $\mu_{T_{C}}$ can be defined by

$$
\mu_{T_{C}}=\sum_{k=k_{\text {start }}}^{\overline{k_{M O}}} p[k] .
$$

Similarly, the average total up time $\mu_{T_{T}}$ in the planed coverage area of the WLAN can be defined as

$$
\mu_{T_{T}}=E\left\{T_{T}\right\}=\sum_{k=k_{\text {start }}}^{k_{\max }} p[k],
$$

Hence the WLAN utilization is

$$
\text { WLAN Utilization }=\frac{\mu_{T_{C}}}{\mu_{T_{T}}} .
$$

Last but not least, our performance evaluation framework considers the user perceived QoS by estimating the user available bandwidth and the Head-of-Line $(\mathrm{HoL})$ packet delay as follows. The MT available bandwidth is calculated as

$$
\begin{aligned}
B W_{A v} & =\frac{\zeta_{L T} R_{W} \tau_{W}+R_{C} \tau_{C}}{\tau_{C}+\tau_{W}} \\
& =\frac{\zeta_{L T} R_{W}\left(\overline{k_{M O}}-k_{\text {start }}\right)+R_{C}\left(k_{\max }-\overline{k_{M O}}\right)}{\left(k_{\max }-k_{\text {start }}\right)},
\end{aligned}
$$

where $\zeta_{L T}$ represents the WLAN usage efficiency as the percentage of the WLAN up duration over the MT WLAN usage duration and can be estimated as $\zeta_{L T}=\sum_{k=k_{\text {sart }}}^{k_{\text {max }}} \overline{P_{M O}}[k] \frac{\sum_{h=1}^{k} p[h]}{k}$, $R_{W}$ and $R_{C}$ are the effective data rates in WLAN and cellular networks respectively, $\tau_{W}$ and $\tau_{C}$ represent the durations spent by the MT within the WLAN and the 3G network respectively.

In order to study the effect of HoL packet delay, we assume a delay threshold for the packet in this hop, which can be considered part of the delay budget of the real-time application packet assigned to the current wireless hop. Consequently, the average packet delay probability $(D)$ can be calculated as

$$
D=\frac{\sum_{k=k_{\text {start }}}^{\overline{k_{M O}}} P_{D}[k]}{\left(\overline{k_{M O}}-k_{\text {start }}+1\right)},
$$

where $P_{D}[k]$ represents the probability that a packet will be delayed more than the predefined delay threshold, which is equal to the probability of a sequence of WLAN down states whose duration is equal to the predefined threshold.

In the next section we present the results obtained from simulation and analysis for a hysteresis-based algorithm [3] and its modified versions ALIVE-HO and LIVE-HO [12].

\section{REsults}

We have simulated the VHO algorithms using MATLAB. The simulation model assumes a MT moving away from the WLAN access point with a constant speed $V$. The $3 \mathrm{G}$ network is assumed to support global coverage. The WLAN parameters are used as in [16] and [12]. Table I shows the values of all the simulation parameters. These parameters result in a WLAN coverage of 100 meters approximately. We compare the performance of a hysteresis-based algorithm [3], ALIVE$\mathrm{HO}$ and LIVE-HO [12]. The hysteresis-based algorithm uses two different thresholds $M I T_{W L A N}$ and $M O T_{W L A N}$ for MI and MO respectively. The MT performs a MI if the $\overline{R S S}[k]$ is larger than $M I T_{W L A N}$ and performs a MO if $\overline{R S S}[k]$ is smaller than the predefined $M O T_{W L A N}$.

In lifetime-based algorithms, The $R S S$ is used to estimate $\overline{R S S}$, an averaged value for $R S S$, and $S[k], R S S$ decay rate. Both parameters are used to estimate the beneficial WLAN availability duration as in (1). Additionally, the estimated lifetime is adapted to the QoS requirements of the active real-time applications by tuning the application specific signal threshold $(A S S T)$ parameter. The MT performs a MO if the averaged received signal strength is less than or equal to a predefined MO threshold, $M O T_{W L A N}$, and the estimated lifetime is less than or equal to a predefined handoff delay threshold, $T_{H O}$, where $T_{H O}$ is set to the expected handoff delay between the two access technologies. We consider ALIVE$\mathrm{HO}$ and LIVE-HO algorithms in our comparison because the algorithm have several parameters that clarify the tradeoff between resource utilizations and the user perceived QoS.

Figure 3 shows the number of handoffs performed by the MT as it moves away from the AP. As shown in this figure, ALIVE-HO and LIVE-HO perform fewer handoffs when compared to the hysteresis-based algorithm. It is well-known 
TABLE I: Simulation parameters values

\begin{tabular}{|l|c||l|l|}
\hline Parameter & Value & Parameter & Value \\
\hline \hline$P_{T}$ & $100 \mathrm{mWatt}$ & $T_{\text {sampling }}$ & $0.01 \mathrm{sec}$ \\
\hline \hline $\mathrm{n}$ & 3.3 & $M O T_{W L A N}$ & $-85 \mathrm{dBm}$ \\
\hline \hline$\sigma$ & $7 \mathrm{~dB}$ & $M I T_{W L A N}$ & $-80 \mathrm{dBm}$ \\
\hline \hline $\mathrm{S}$ & $28.7 \mathrm{~dB}$ & $T_{\text {handoff }}$ & $1 \mathrm{sec}$ \\
\hline \hline$D_{\text {average }}$ & $0.5 \mathrm{~m}$ & $R_{W}$ & $25 \mathrm{Mbps}$ \\
\hline \hline$D_{\text {slope }}$ & $5 \mathrm{~m}$ & $R_{C}$ & $2.4 \mathrm{Mbps}$ \\
\hline$\alpha$ & $-90 \mathrm{dBm}$ & & \\
\hline
\end{tabular}

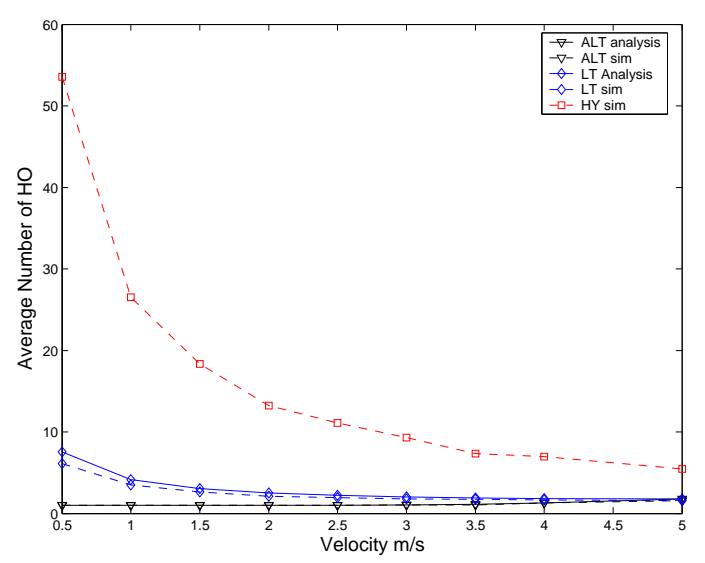

Fig. 3: Number of handoffs $($ ASST $=-90 \mathrm{dBm})$

that unnecessary handoffs may overload the network resulting in severe degradation in the overall performance. ALIVE-HO optimally performs by making approximately one MO near the WLAN edge at the cost of introducing a velocity estimator. In addition, this figure also shows good match between the simulation and the analytical results reinforcing the correctness of the performance evaluation framework.

Figure 4 shows the MT WLAN utilization in the transition region as defined in the previous section. The Figure shows again the superiority of ALIVE-HO and LIVE-HO, which results from extending the WLAN MO distance. This increment

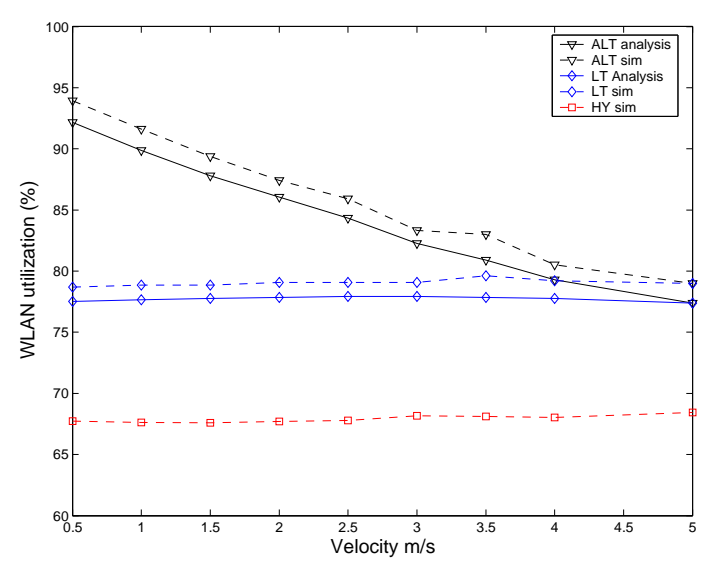

Fig. 4: WLAN utilization $($ ASST $=-90 \mathrm{dBm})$.

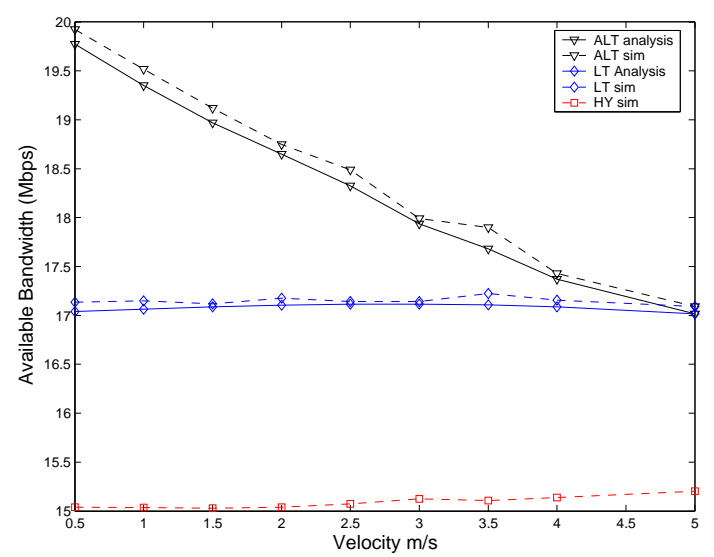

Fig. 5: MT available bandwidth (ASST $=-90 \mathrm{dBm})$

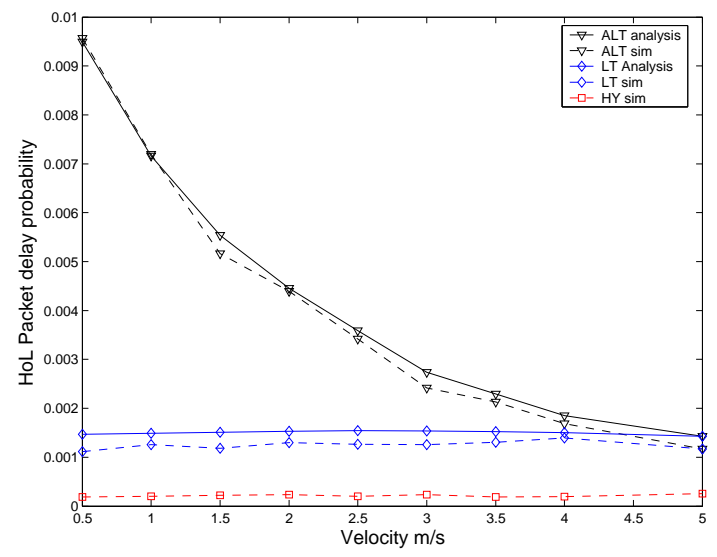

Fig. 6: HoL packet delay rate (ASST $=-90 \mathrm{dBm}$, Delay threshold $=30 \mathrm{~ms}$ )

in WLAN utilization means that extending WLAN utilization beneficially is possible despite of the channel degradation near the WLAN edge. Furthermore, the figure shows a good match between the simulation and the analytical results.

However, extending the lifetime of the MT within the WLAN result in a tradeoff between different QoS metrics. On the one hand, it improves the user available bandwidth as shown in Figure 5. Consequently, it is preferable to perform MO handoff only once at the WLAN edge especially at low mobility. On the other hand, extending the WLAN utilization increases the packets delay probability due to channel condition degradation. As shown in Figure 6, the HoL packet delay probability using ALIVE-HO and LIVE-HO is larger than that resulting from using the traditional hysteresis algorithm. Hence, a handoff algorithm designer should consider this common tradeoff between the MT throughput and packet delay, especially with the increasing interest in bandwidthconsuming multimedia applications. In [12], the authors proposed tuning the ASST to realize better resource utilization as well as achieving satisfactory QoS for the active realtime applications. Figure 7$]$ and Figure 8 show the impact of ASST variation on the available bandwidth and the HoL packet 


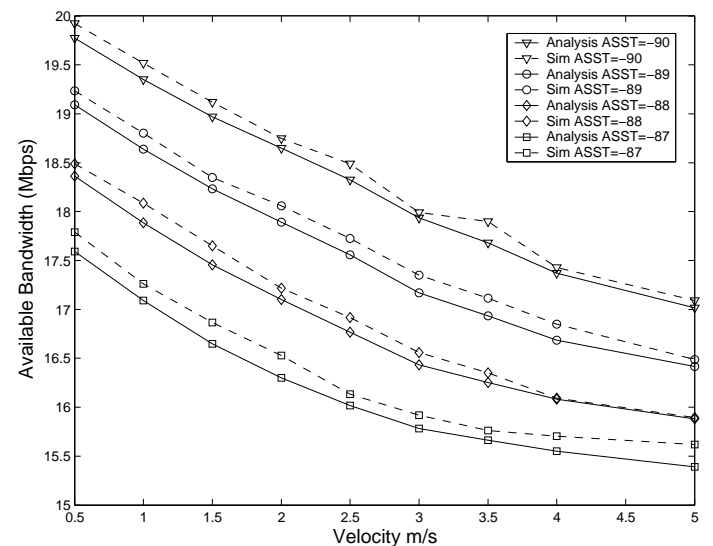

Fig. 7: User available bandwidth for different ASSTs

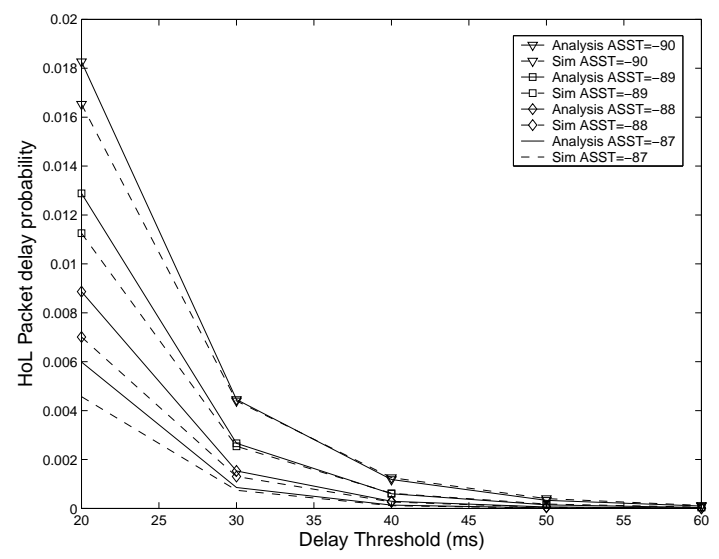

Fig. 8: HoL packet delay rate $(\mathrm{V}=1 \mathrm{~m} / \mathrm{s})$

delay probability respectively. As shown from the figures, by decreasing the ASST, one can obtain smaller packet delay probability at the cost of decreasing the available bandwidth.

\section{Conclusions}

The convergence of wireless access technologies toward 4G wireless networks should overcome several challenges before practical implementation. One of the major challenges is user mobility handling between different access technologies in order to keep the user connected to the best available network. This network is usually the underlay network that can provide better service at lower cost to the user, as well as improve the overall system resource utilization. However, achieving both goals requires a well designed handoff algorithm that can compromise the tradeoff between efficient resource utilization and user perceived QoS. In this paper, we present a framework that can be used to compare the performance of different vertical handoff algorithms. In addition we compare the performance of different algorithms using simulation and analysis demonstrating a good match between them. Furthermore, our results provide a quantitative means to evaluate the critical impact of the handoff algorithm design on satisfying the active real-time application requirements while improving the overall resource utilization.

\section{REFERENCES}

[1] E. Gustafsson and A. Jonsson, "Always best connected," IEEE Wireless Communications, vol. 10, no. 1, pp. 49 - 55, Feb. 2003.

[2] M. Stemm and R. H. Katz, "Vertical handoffs in wireless overlay networks," ACM Mobile Networks and Applications, vol. 3, no. 4, pp. $335-350,1998$.

[3] M. M. Buddhikot, G. Chandranmenon, S. Han, Y. W. Lee, S. Miller, and L. Salgarelli, "Integration of 802.11 and third generation wireless data networks," in Proc. of IEEE INFOCOM, San Francisco, US, Apr. 2003, pp. $503-512$.

[4] R. Chakravorty, P. Vidales, K. Subramanian, I. Pratt, and J. Crowcroft, "Performance issues with vertical handovers - experiences from GPRS cellular and WLAN hot-spots integration," in Proc. of the Second IEEE Annual Conference on Pervasive Computing and Communications, Mar. $14-17$ 2004, pp. $155-164$.

[5] S. Y. Hui and K. H. Yeung, "Challenges in the migration to $4 \mathrm{G}$ mobile systems," IEEE Communications Magazine, vol. 41, no. 12, pp. 54-59, Dec. 2003.

[6] A. K. Salkintzis, C. Fors, and R. Pazhyannur, "WLAN-GPRS integration for next-generation mobile data networks," IEEE Wireless Commun., vol. 9, no. 5, pp. 112 - 124, Oct. 2002.

[7] J. Makela, M. Ylianttila, and K. Pahlavan, "Handoff decision in multiservice networks," in Proc. of 11th IEEE International Symposium on Personal, Indoor and Mobile Radio Communications (PIMRC'00), vol. 1, London, UK, Sep. 2000, pp. 655 - 659 .

[8] K. Pahlavan, P. Krishnamurthy, A. Hatami, M. Ylianttila, J. P. Makela, R. Pichna, and J. Vallstron, "Handoff in hybrid mobile data networks," IEEE Commun. Mag., vol. 7, no. 4, pp. 34 - 47, Apr. 2000.

[9] H. Wang, R. H. Katz, and J. Giese, "Policy-enabled handoffs across heterogeneous wireless networks," in Proc. of the Second IEEE Workshop on Mobile Computer Systems and Applications, New Orleans, Louisiana, Feb. 1999, p. 51.

[10] A. Hatami, P. Krishnamurthy, K. Pahlavan, M. Ylianttila, J. Makela, and R. Pichna, "Analytical framework for handoff in non-homogeneous mobile data networks," in Proc. of (PIMRC'99), Osaka, Japan,, Sep. 1999 , pp. $760-764$.

[11] M. Ylianttila, J. Makela, and P. Mahonen, "Supporting resource allocation with vertical handoffs in multiple radio network environment," in Proc. of IEEE International Symposium on Personal, Indoor and Mobile Radio Com-munications (PIMRC'02), Lisbon, Portugal, Sep. 2002, pp. $64-68$.

[12] A. H. Zahran and B. Liang, "ALIVE-HO: Adaptive lifetime vertical handoff for heterogeneous wireless networks," Technical Report, University of Toronto.

[13] T. S. Rappaport, Wireless Communications: Principles and Practice. Prentice Hall, July 1999.

[14] N. Zhang and J. M. Holtzman, "Analysis of handoff algorithms using both absolute and relative measurements," IEEE Transactions on Vehicular Technology, vol. 45, no. 1, pp. 174 - 179, Feb. 1996.

[15] G. Bolch, S. Greiner, H. de Meer, and K. S. Trivedi, Queuing networks and Markov Chains: Modeling and Performance Evaluation with Computer Science Applications, 2nd ed. Wiley, August 1998.

[16] A. Majlesi and B. H. Khalaj, "An adaptive fuzzy logic based handoff algorithm for hybrid networks," in Proc. of 6th International Conference on Signal Processing, vol. 2, Aug. 2002, pp. 1223 - 1228. 